REVISIÓN DE LITERATURA

\title{
PRODUCCIÓN DE ÁCIDO LÁCTICO POR VÍA BIOTECNOLÓGICA
}

\section{BIOTECHNOLOGICAL PRODUCTION OF LACTIC ACID}

\author{
Carlos A. Garcia ${ }^{1}$, Guillermo S. Arrázola², Alba M. Durango ${ }^{3}$ \\ Recibido para evaluación: Mayo 20 de 2010 - Aceptado para publicación: Noviembre 30 de 2010
}

\begin{abstract}
RESUMEN
El ácido láctico es un ácido orgánico valorado por su aplicación en la industria de alimentos, farmacéutica, química y su potencial como materia prima para la producción de polímeros biodegradables. Por lo tanto, la producción biotecnológica de ácido láctico ha adquirido gran importancia industrial con respecto a la síntesis química debido a que usa materias primas renovables y es amigable con el ambiente. En décadas recientes las investigaciones están direccionadas a optimizar la producción de ácido láctico con la premisa de lograr mayor productividad, rendimientos y bajo costo. Los parámetros del bioproceso tales como los microorganismos, la composición de los nutrientes, los sistemas de producción, bioreactores y metodologías de análisis son revisadas en este documento.
\end{abstract}

Palabras clave: Biotecnología, subproductos alimenticios, ácido láctico.

\begin{abstract}
Lactic acid is an organic acid valued for its application in the food, pharmaceutical and chemical industries and its potential as raw material for the production of biodegradable polymers. Biotechnological production of lactic acid has acquired great industrial importance with regard to the chemical synthesis since using renewable raw materials and is friendly with the environment. In recent decades investigations are addressed to optimize the production of lactic acid with the premise of achieving higher productivity, performance and low cost. Parameters of the bioprocess such as microorganisms, nutrient composition, production, bioreactor, and methodologies of analysis systems are reviewed in this document.
\end{abstract}

Keywords: Biotechnology, food by-products, lactic acid.

\footnotetext{
${ }^{1}$ Ingeniero de Alimentos, Estudiante de Maestría en Ciencias Agroalimentarias, Departamento de Ingeniería de Alimentos, Universidad de Córdoba, Montería, Colombia

${ }^{2}$ Ingeniero de Alimentos Ph.D. Profesor titular, Departamento de Ingeniería de Alimentos, Universidad de Córdoba, Montería, Colombia.

${ }^{3}$ Bacterióloga Ph.D. Profesor titular, Departamento de Ingeniería de Alimentos, Universidad de Córdoba, Montería, Colombia. adurango@sinu.unicordoba.edu.co, durangoalba@yahoo.com
} 


\section{INTRODUCCIÓN}

La producción biotecnológica del ácido láctico ha adquirido gran importancia debido a los beneficios ambientales y al uso de recursos renovables en lugar de los petroquímicos. Hay numerosas investigaciones sobre el desarrollo de métodos biotecnológicos para la producción de ácido láctico, con el objetivo de hacer el proceso más eficiente y económico. El ácido láctico es un ácido orgánico natural de importancia industrial en las aplicaciones farmacéuticas como electrolito y fuente de minerales; en la industria cosmética como $\mathrm{pH}$ buffer, antimicrobiano y rejuvenecedor de la piel; como neutralizante, solvente $y$ agente limpiador en la industria química $y$ en la industria alimentaria como acidulante, preservante y antimicrobiano (Gündüz 2005), y se utiliza en una gran variedad de alimentos procesados como caramelos, productos de panadería, sopas, lácteos, cerveza, jaleas, mermeladas, mayonesas y huevos procesados (Rojan et al. 2007). Existen dos formas ópticas activas, donde el ácido $L(+)$ láctico es el preferido. La aplicación de procesos de separación, concentración y secado permiten obtener ácido $L(+)$ láctico purificado cuya demanda mundial se espera para el 2011 sea superior a las 200.000 toneladas métricas (Gopal et al. 2008) de las cuales el $85 \%$ de la demanda son aplicaciones en alimentos y el $15 \%$ restante en la industria no alimentaria (Rojan et al. 2009). En Colombia todo el producto se importa y equivale a mil millones de pesos anuales (Serrato y Caicedo 2005). Nuevas aplicaciones de ácido L (+) láctico, tales como monómeros o plásticos biodegradables (PLA) tienen un gran potencial de expansión en el mercado, de tal forma que la demanda mundial estaría alrededor del 14 al 19\% (Gopal et al. 2008).

La producción biotecnológica de ácido láctico ofrece varias ventajas como el bajo costo de los sustratos, bajas temperaturas de producción y bajo consumo de energía (Rojan et al. 2007). Las recientes investigaciones están enfocadas en encontrar nuevas y efectivas fuentes nutricionales y nuevas técnicas de fermentación encaminadas a alcanzar altas conversiones de sustrato y altos rendimientos en ácido láctico (Sule et al. 2004). Esta revisión se enfoca en las distintas tecnologías de fermentación para producir ácido láctico a partir de los microorganismos, las materias primas renovables, los sistemas de fermentación evaluados y las herramientas estadísticas de los análisis usadas para optimizar el proceso biotecnológico de producción de éste.

\section{PRODUCCIÓN BIOTECNOLÓGICA DE ÁCIDO LÁCTICO}

El ácido láctico se denomina ácido 2-hidroxipropanoico y está formado por los grupos funcionales alcohol y carboxilo, conformando un carbono asimétrico que le confiere su actividad óptica. Existen dos isómeros ópticos, el D(-) láctico y el L(+) láctico (Figura 1) y una forma racémica constituida por fracciones equimolares de las formas L(+) y D(-) (Gopal et al. 2008; Vijayakumar et al. 2008). El isómero $D(-)$ es perjudicial al metabolismo humano y puede generar acidosis y descalcificación (Panesar et al. 2007). El ácido $\mathrm{L}(+)$ láctico es clasificado por la FDA como una sustancia GRAS, generalmente reconocido como seguro para uso como aditivo alimenticio (Vijayakumar et al. 2008; Rojan et al. 2009). 
<smiles>C[C@H](O)C(=O)O</smiles>

L(+) ácido láctico<smiles>C[C@H](O)C(=O)O</smiles>

$\mathrm{D}(-)$ ácido láctico
Figura 1. Estructuras isoméricas del ácido láctico

Tanto las dos formas ópticamente activas como la racémica se encuentran en formas líquidas, incoloras y solubles en el agua. En estado puro son sólidos altamente higroscópicos de punto de fusión bajo. Las formas isoméricas del ácido láctico pueden ser polimerizadas y producidas con diferentes propiedades (Serna y Rodriguez 2005). Los isómeros puros del ácido láctico pueden ser obtenidos por fermentación microbiana a partir de una fuente renovable.

La producción biotecnológica está basada en la fermentación de sustratos ricos en carbohidratos (Figura 2) por medio de bacterias u hongos para formar los enantiómeros ópticamente activos y depende del tipo de microorganismo utilizado, la inmovilización o recirculación del microorganismo, el pH, la temperatura, la fuente de carbono, la fuente

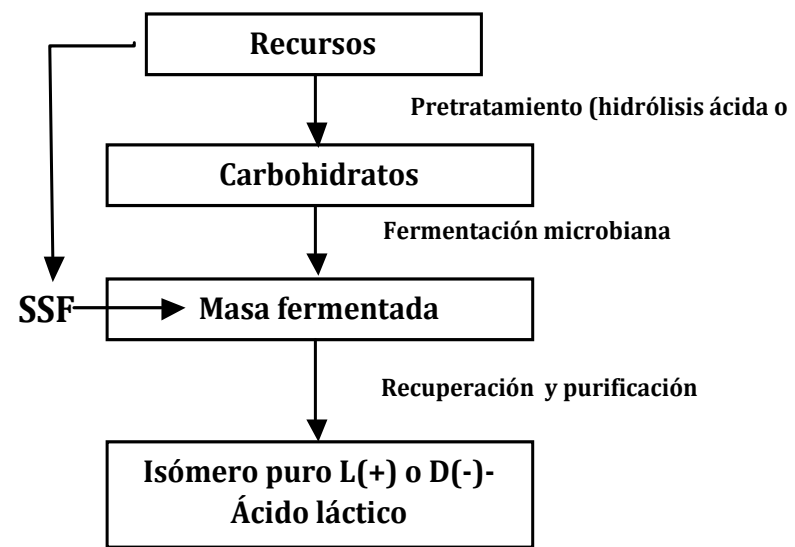

Figura 2. Manufactura de ácido láctico por fermentación microbiana por simultánea sacarificación y fermentación(SSF) de nitrógeno, el método de fermentación empleado y la formación de los subproductos (Hofvendahl y Hagerdal 2000). Dependiente del tipo de carbohidrato, el proceso requerirá de una etapa que convierta las materias primas renovables a sustratos fermentables, sea ésta por degradación enzimática, química o por adaptación metabólica del microorganismo.

\section{MICROORGANISMOS UTILIZADOS EN LA PRODUCCIÓN DE ÁCIDO LÁCTICO}

La mayoría de las investigaciones se han llevado a cabo con las Bacterias Ácido Lácticas (BAL) y los hongos filamentosos del género Rhizopus. Según Zhang et al. (2007a), el uso de los hongos tiene ventaja por las características amilolíticas y bajos requerimientos en nutrientes.

La morfología de los hongos, es una característica de interés en la producción del ácido,porque puede influir positiva como negativamente; los hongos pueden crecer en forma de micelio individual, granular, en flóculos en pellets esféricos. Marták et al. (2003) encontraron que cuando la morfología de $R$. arrhizus cambió de filamentosa a pellets, después de $152 \mathrm{~h}$ decreció la productividad del ácido láctico de 75,3\% a 62,6\%. En un cultivo de $R$. oryzae la producción de ácido láctico fue mejorada al ser inducido a la forma micelial (Park et al.1998; Yu et al. 2007). Dong-Mei et al. (2003) estudiaron los factores que afectan la morfología y productividad de $R$. oryzae $R 1021$ y determinaron que la forma de pellet exhibe un mayor rendimiento de ácido láctico que la forma flocular. Zhang et al. (2007a) reportaron que es favorable inducir la morfología de pellets pequeños y sueltos de 
Rhizopus spp., en condiciones específicas de la producción de ácido láctico.

La fermentación fúngica tiene la ventaja de requerir un medio simple para producir ácido láctico, pero también tiene requerimientos elevados de aireación. Haung et al. (2005) produjeron ácido láctico a partir de aguas residuales que contenían fécula de patata utilizando $R$. oryzae y $R$. arrhizus. Tay y Yang (2002) inmovilizaron $R$. oryzae en un lecho fibroso con glucosa y almidón para producir ácido láctico. En muchos casos la glucosa fue preferida como fuente de carbono para Rhizopus spp., seguido del almidón (Zhang et al. 2007b).

Cuando se utilizan las bacterias, se busca que éstas sean preferiblemente termófilas, que fermenten rápida y completamente los sustratos baratos, con adición mínima de nutrientes nitrogenados, que crezcan en valores bajos de $\mathrm{pH}$, que presenten poca producción de biomasa y una despreciable cantidad de subproductos. Los géneros Lactobacillus, Streptococcus, Enterococcus son los más estudiados en la producción. Rivas et al. (2004b) produjeron ácido láctico de la mazorca del maíz por sacarificación y fermentación simultánea utilizando Lactobacillus rhamnosus. Wee et al. (2006a) informaron la producción económica de ácido $L(+)$ láctico de melaza de azúcar por fermentación batch con Enterococcus faecalis. Kourkoutas et al. (2005) utilizaron para la producción del ácido láctico Lactobacillus casei inmovilizado en trozos de frutas.

Kenji et al. (2007) informaron de la producción de ácido $\mathrm{L}(+)$ láctico a partir de almidón nativo con Streptococcus bovis 148. Rojan et al.(2006) estudiaron la fermentación en estado sólido a partir de residuos de los productos agrícolas utilizando Lactobacillus delbrueckii. Nancib et al. (2005) explicaron el efecto conjunto de fuentes de nitrógeno y la administración de suplementos de vitamina $B$ en jugos por Lactobacillus casei subsp. rhamnosus. Ohkouchi y Inoue (2006) estudiaron la producción directa de ácido $\mathrm{L}(+)$ láctico a partir de almidón y desechos de alimentos utilizando Lactobacillus manihotivorans LMG 18011; Vasala et al. (2005) utilizaron productos lácteos con alto contenido de sal utilizando Lactobacillus salivarius ssp. salicinius.

Plessas et al. (2008) utilizaron cultivos mixtos de Kluyveromyces marxianus (IFO 288), Lactobacillus delbrueckii ssp. bulgaricus (ATCC 11842) y Lactobacillus helveticus (ATCC 15009), logrando la mayor concentración cuando se mezclaron los dos primeros; $\mathrm{Xu}$ et al. (2006) trabajaron con Lactobacillus sake y Lactobacillus casei a partir de hidrolizado de soya. Por proliferación selectiva, Sakai et al. (2007) informaron el uso de Lactobacillus plantarum a partir de la fermentación de desperdicios de cocina y Bustos et al. (2004) utilizaron Lactobacillus pentosus a partir de podas de sarmientos y lías de vinificación.

Otras bacterias de importancia industrial incluyen Lactobacillus delbruickii ssp bulgaricus, que utiliza la lactosa como fuente de carbono y Lactobacillus pentosus, que puede utilizar las pentosas de sulfito de residuos de licor. Otras especies homofermentativas son Lactobacillus casei, Lactobacillus leichmanii, Streptococcus lactis y Lactococcus lactis (Ohashi et al. 1999).

Para mejorar la productividad de ácido 
láctico se han modificado genéticamente microorganismos, tales como Lactobacillus lactis NCIM 2368 (Joshi et al. 2010), Lactobacillus delbrueckii NCIM 2365 (Patil et al. 2006; Pessôa de França et al. 2009), Lactobacillus delbrueckii Mutant Uc-3 (Adsulet al. 2007; Dumbrepatil et al. 2008), Lactobacillus helveticus CNRZ32 (Kyla“-nikkila et al. 2000) y P. stipitis (Marja Ilmén et al. 2007).

\section{Características de las bacterias ácido lácticas}

Son Gram positivos, microaerofílicos y catalasa negativos, forman ácido láctico como producto principal de la fermentación de los azúcares y pueden ser homofermentativos o heterofermentativos según la cantidad y la presencia del ácido. Existen bacterias homofermentativas obligadas y facultativas; dando lugar al ácido láctico como producto principal de la fermentación. Este grupo está integrado por Lb. caucasicus, Lb. bulgaricus, Lb. lactis, $L b$. helveticus, $\mathrm{Lb}$ acidophilus y $\mathrm{Lb}$. delbrueckii.
La mayoría de las especies pertenecientes a estos géneros tienen alta tolerancia a $\mathrm{pH}$ por debajo de 5,0, lo que les da la ventaja competitiva sobre otras bacterias; la temperatura optima de crecimiento ésta en un rango de $20^{\circ} \mathrm{C}$ a $40^{\circ} \mathrm{C}$ y varía entre géneros (Hofvendahl y Hagerdal 2000). Son microorganismos de rápido crecimiento con pequeños genomas, metabolismo simple y relevancia industrial. La mayoría de los Lactobacillus producen únicamente una forma isomérica de ácido láctico; las formas isoméricas de lactato deshidrogenasa presente en Lactobacillus determinan el isómero de ácido láctico producido, ya que la deshidrogenasa láctica es esteroespecífica; las especies de los géneros Aerococcus, Carnobacterium, Enterococcus, Vagococcus y Tetragenococcus producen únicamente isómeros $D$ (Tabla 1). Sin embargo, algunos Lactobacillus producen formas racémicas donde el isómero predominante depende de los cambios en la aireación, la cantidad de $\mathrm{NaCl}$, el tipo de fermentación, el incremento de $\mathrm{pH}$ y la concentración de

Tabla 1. Bacterias ácido lácticas homo y heterofermentativas y la configuración

\begin{tabular}{lccc}
\hline Género y especie & Homofermentativa & Heterofermentativa & Configuración ácido láctico \\
\hline Lactobacillus & & & $\mathrm{D}(-)$ \\
L. delbrueckii & + & - & $\mathrm{D}(-)$ \\
L. lactis & + & - & $\mathrm{D}(-)$ \\
L. bulgaricus & + & - & $\mathrm{L}(+)$ \\
L. casei & + & - & $\mathrm{DL}$ \\
L. plantarum & + & - & $\mathrm{DL}$ \\
L. curvatus & + & - & $\mathrm{DL}$ \\
L. brevis & - & + & $\mathrm{DL}$ \\
L. fermentum & - & + & \\
Sporolactobacillus & & & $\mathrm{D}(-)$ \\
S. inulinus & + & - & \\
Streptococcus & & & $\mathrm{L}(+)$ \\
S. cremoris & + & - & $\mathrm{L}(+)$ \\
S. lactis & + & - & $\mathrm{D}(-)$ \\
Leuconostoc & & & \\
L. mesenteroides & - & + & \\
\hline
\end{tabular}


sustrato. Las especies del género Lactobacillus producen además de las formas isoméricas $\mathrm{L}(+)$ y $\mathrm{D}(-)$, una mezcla racémica de ambos isómeros.

Las homofermentativas usualmente metabolizan la glucosa por la vía EmbdenMeyerhof, dando como resultado dos moléculas de ácido láctico de cada molécula de glucosa con un rendimiento mayor a 0,9 $\mathrm{gg}^{-1}$. Solamente las bacterias ácido lácticas homofermentativas están disponibles para la producción comercial de ácido láctico (Hofvendahl y Hagerdal 2000).

La elección de un microorganismo depende del carbohidrato a ser fermentado: Lactobacillus delbreuckii ssp. delbreuckii fermentan la sacarosa; Lactobacillus delbreuckii ssp. bulgaricusus a la lactosa; Lactobacillus helveticus es capaz de fermentar lactosa y galactosa; Lactobacillus amylophylus y Lactobacillus amylovirus fermentan almidón; Lactobacillus lactis puede fermentar glucosa, sacarosa y galactosa, Lactobacillus pentosus puede fermentar licores ricos en sulfitos (Okafor 2007).

El estudio de la producción de ácido láctico cultivando células en un medio con diferentes azúcares reveló que Lactobacillus casei prefiere la lactosa para su crecimiento y la producción de ácido láctico, seguido por glucosa y maltosa; mientras la sacarosa fue poco usada (Narayanan et al. 2004). Entre las principales BAL amilolíticas reportadas para la producción de ácido láctico se encuentran $L$. amylophilus (Altaf et al. 2005; 2006; 2007; Naveena et al. 2005b), Lactobacillus spp. (Wang et al. 2005), Lactococcus lactis (Okano et al. 2007), L. plantarum y Leuconostoc St3-28 (Thomsen et al. 2007).

\section{MATERIAS PRIMAS PARA LA PRODUCCIÓN BIOTECNOLÓGICA DE ÁCIDO LÁCTICO}

Los medios de cultivo son una mezcla de nutrientes que en las concentraciones adecuadas y en las condiciones físicas óptimas, permiten el crecimiento de los microorganismos. Sin embargo, para que las bacterias crezcan adecuadamente en un medio de cultivo deben reunir una serie de condiciones como la temperatura, el grado de humedad y la presión de oxígeno adecuado, así, como un grado correcto de acidez o alcalinidad. Por lo tanto, un medio de cultivo debe contener los nutrientes y los factores de crecimiento necesarios y estar exento de todo microorganismo contaminante.

Han sido usados diferentes sustratos para la producción fermentativa de ácido láctico con Lactobacillus; así, el producto más puro ha sido obtenido cuando se usa un medio simple, resultando en menos costos de purificación (Panesar et al. 2007b). Hay varios factores que estimulan el crecimiento y que tienen considerable efecto sobre la producción de ácido láctico. La mezcla de aminoácidos y péptidos usualmente estimulan el crecimiento de las BAL y resultan en velocidades de crecimiento mucho más altas que en un medio libre de aminoácidos. Vázquez (2008) evaluó el efecto de peptonas de diversas fuentes en los medios de cultivo para BAL y ninguna de ellas maximizó el crecimiento. Costa et al. (2008) utilizaron un medio con $1 \% \mathrm{p} / \mathrm{v}$ de glucosa, $1 \% \mathrm{p} / \mathrm{v}$ de fructosa, $1 \%$ de extracto de levadura 
y minerales ( $\mathrm{K}, \mathrm{Mg}$ y $\mathrm{Mn}$ ) para evaluar la producción de los isómeros de ácido láctico a partir de cepas de Lactobacillus aisladas de una fermentación alcohólica. Serna y Rodríguez (2005) variaron la concentración de glucosa entre 20, 60 y $110 \mathrm{~g} \mathrm{~L}^{-1}$ en MRS para evaluar el potencial de la cepa de Lactococcus lactis ssp lactis aislada de plantas de caña de azúcar; Lee (2004) suplementó MRS con $20 \mathrm{~g} \mathrm{~L}^{-1}$ glucosa en cultivo mixto de $L b$. casei; $L b$. delbrueckkii ssp. lactis, Lb. helveticus, Lb. delbrueckii. MinTian et al. (2005) utilizaron un medio de $100 \mathrm{~g}$ $\mathrm{L}^{-1}$ de glucosa y Serrato et al. (2005) utilizaron un medio compuesto por sacarosa $\left(45 \mathrm{~g} \mathrm{~L}^{-1}\right) \sin$ refinar y extracto de levadura $\left(15 \mathrm{~g} \mathrm{~L}^{-1}\right)$.

La viabilidad de la producción biotecnológica de ácido láctico está sujeta a que las materias primas tengan las siguientes características: baratas, bajos niveles de contaminantes, rápidas tasa de producción, alto rendimiento, subproductos formados, capacidad para ser fermentado con poco o ningún pre-tratamiento y disponibilidad durante todo el año. Se han usado materias primas baratas para la producción económica del ácido láctico, entre estos materiales están los celulósicos, los amiláceos, el lactosuero y la melaza.

Los amiláceos son fuente de polisacáridos (principalmentealmidón) baratos, abundantes y renovables. Los materiales amiláceos utilizados incluyen el sorgo (4,08\% almidón), la paja de trigo (54\% almidón), el maíz, la yuca (27\% almidón), el ñame, la papa (Liu et al. 2005), el arroz y la cebada (Oh et al. 2005). Sin embargo, estos materiales deben ser hidrolizados hasta azúcares fermentables antes del proceso porque están formados por enlaces de glucosa $\alpha(1,4)$ y $\alpha(1,6)$, aunque puede Ilevarse a cabo simultáneamente con la fermentación. Wee et al. 2005) produjeron ácido láctico a partir de hidrolizados de almidón de diferentes fuentes y concentraciones con Enterococcus faecalis RKY1. En investigaciones con bagazo de yuca se obtuvieron rendimientos de 0,90 a 0,98 $\mathrm{gg}^{-1}$ usando L. delbrueckii NCIM 2025 y L. casei (Rojan et al. 2005; Rojan et al. 2006a,b,c; Rojan et al. 2007), yuca en polvo (Limin et al. 2010b). Rendimientos similares se obtuvieron utilizando almidón de papa y maíz como materia prima (Zhang et al. 2005; Nagarjun et al. 2005; Altaf et al. 2007; Rojan et al. 2009). Altaf et al. (2007) reportó el uso de almidon crudo de harinas suplementado con levaduras de panaderia.

Otra materia prima muy utilizada es la melaza que contiene sacarosa $(31 \% \mathrm{p} / \mathrm{v})$, glucosa $(9,5 \% \mathrm{p} / \mathrm{v})$, fructosa $(10 \%)$ y nitrógeno $(0,95 \%)$ y es un sustrato completo para la producción de ácido láctico (Dumbrepatil et al. 2008; Patil et al.2006; Wee et al. 2006b; Bhatta y Srivastava 2008; Pessôa de França et al. 2009).

Los lignocelulósicos consisten en celulosa, hemicelulosa y lignina. La primera es una cadena lineal de $\beta$ D-glucanos, la segunda es un heteropolisacárido que al hidrolizarlo incluye xilosa, glucosa, manosa, arabinosa, galactosa y otros azúcares dependiendo del origen (Zhang et al. 2007a). Como materiales lignocelulósicos (MLC) con gran potencial como materia prima están el sarmiento de vid (Bustos et al. 2004), jugos de cogollo, hojas y jugo de caña de azúcar (Serna y Rodríguez 2006; 2007), Salvado de trigo (Naveena et al. 2005a; Naveena et al. 2005b), la paja de arroz (Qi y Yao 2007), el salvado de arroz (Tanaka et al. 2005), los granos agotados de cervecería (Mussatto et al. 2008). 
El lactosuero es uno de los principales subproducto de la industria láctea, contiene aproximadamente $4,85 \% \mathrm{p} / \mathrm{v}$ de lactosa, 0,8\% p/v de proteína, 0,5\% de grasa, 0,6\% de minerales y vitaminas, y $0,05 \%$ de ácido láctico (Altiok 2004). Los dos tipos comunes de suero son el dulce y el ácido (Panesar et al. 2007a) exitosamente usados en la producción de ácido láctico (Gündüz 2005; Panesar et al. 2007b; Plessas et al. 2008), suplementado con extracto de levadura (Arasaratnam et al. 1996; Schepers et al. 2002; Göksungur, 2005) y con glucosa (Mirdamadi et al. 2008).

Para la producción de ácido láctico también han sido utilizados desechos orgánicos derivados del procesamiento de alimentos como los residuos del procesamiento de zanahoria, bagazo y lodos de caña (Pandey 2006), almidón y desechos de alimentos (Ohkouchi y Inoue 2006), mazorcas de maíz (Rivas et al. 2004a; 2004b), desechos de manzana (Alonso et al. 2009), desechos de piña (Idris 2006), mucílago de café (Arias et al. 2009), melaza derivada de la producción de xilosa (Shen y Xia 2006; Limin et al. 2010a), fuentes de nitrógeno orgánico (Altaf et al. 2005).

El licor de maíz es un subproducto de la fabricación de almidón de maíz y su composición típica contiene lactosa 3,0 a $4,0 \% \mathrm{p} / \mathrm{v}$, glucosa $0,5 \% \mathrm{p} / \mathrm{v}$, no reductores (principalmente almidón) 1,5\% $\mathrm{p} / \mathrm{v}$, ácido acético $0,05 \% \mathrm{p} / \mathrm{v}$, ácido láctico de glucosa $0,5 \% \mathrm{p} / \mathrm{v}$, feniletilamina $0,05 \% \mathrm{p} / \mathrm{v}$, aminoácidos (péptidos, aminas) 0,5\% p/v, sólidos totales de 80 a $90 \%$ p/v y nitrógeno 0,15 a $0,2 \%$ p/v (Okafor, 2007). El licor de maíz ha sido usado como fuente de nitrógeno (Wee et al. 2006b; Agarwal et al. 2008).

\section{FERMENTACIÓN ÁCIDO LÁCTICA}

Las técnicas de producción de ácido láctico consisten en usar células libres y células inmovilizadas, con el sistema de células libres usando fermentación batch se obtuvieron productividades de $3,97 \mathrm{~g} \mathrm{~L}^{-1} \mathrm{~h}^{-1}$ (Büyü kkileci y Harsa 2004), 3,6 $\mathrm{g} \mathrm{L}^{-1} \mathrm{~h}^{-1}$ (Oh et al. 2005). Diferentes estrategias de fermentación fed -batch para producir ácido láctico fueron Ilevadas a cabo por Ding y Tan (2006), cuando utilizaron glucosa (850 $\mathrm{g} \mathrm{L}^{-1}$ ) y extracto de levadura (1\%) fue el método más efectivo, obteniendo la máxima concentración de ácido L (+) láctico (90,3\%), biomasa $\left(4,30 \mathrm{~g} \mathrm{~L}^{-1}\right)$ y productividad $\left(2,14 \mathrm{~g} \mathrm{~L}^{-1} \mathrm{~h}^{-1}\right)$. Comparado con la fermentación batch este método mostró un $56,5 \%$ p/v de mejoramiento en la producción de ácido láctico. Estudios similares con estrategias de fermentación batch han sido evaluadas por Limin et al. (2010b) quienes investigaron la sacarificación y fermentación simultánea, la fermentación en dos etapas y la liquefaccción.

La fermentación continua tiene la ventaja de lograr altas productividades y no requerir fermentadores grandes (Panesar et al. 2007b). En un cultivo de Lactobacillus helveticus con lactosuero desproteinizado obtuvieron la máxima concentración de ácido láctico $\left(11 \mathrm{Kg} \mathrm{m}^{-3}\right)$ a una tasa de dilución (D) de 0,1 h-1 (Urríbarrí et al. 2004). Schepers et al. (2002) obtuvieron una productividad de ácido láctico de $13,5 \mathrm{~g} \mathrm{~L}^{1} \mathrm{~h}^{-1}$ con $1 \mathrm{~g} \mathrm{L^{1 }}$ de azúcar residual a una $D=0,27 \mathrm{~h}^{-1}$ con inmovilización de Lactobacillus helveticus en un proceso de dos etapas, usando un medio de lactosuero permeado y extracto de levadura. Otras investigaciones usando células inmovilizadas 
de Lactobacillus casei empleando como matriz de inmovilizacion cáscara de huevo, chips de madera (Nabi y Ardalan, 2004), piezas de manzana (Kourkoutas et al. 2005), alginato-quitosano (Göksungur et al. 2005), $\kappa$-Carragenina (Schepers et al. 2006) y gel de pectato (Panesar et al. 2007a).

El ácido láctico producido por fermentación en estado sólido (SSF), es un proceso definido como el crecimiento de microorganismos (principalmente hongos) en material sólido húmedo en ausencia de flujo de agua (MooYoung et al. 1983; Pandey 1992; Gopal et al. 2008), esto favorece considerablemente la reducción de efluentes y por lo tanto, la contaminación. Es un proceso simple, que usa volúmenes pequeños y altas concentraciones de producto y de fácil aireación. Entre los materiales usados como soporte se encuentran el salvado de trigo (Naveena et al. 2005b), torta de cacahuete desgrasada (Nagarjun et al. 2005), paja de arroz (Qi y Yao 2007). Las cepas bacterianas usadas son: Enterococcus faecalis RKY1 (Oh et al.2005), Lactobacillus amylophilus GV6 (Altaf et al. 2005), Lactobacillus amylovorus NRRL B-4542 (Nagarjun et al. 2005), Lactobacillus delbrueckii (Rojan et al. 2006c).

En cuanto a los hongos filamentosos, Huang et al. (2005) utilizaron especies Rhizopus arrhizus 36017 y Rhizopus oryzae 2062 con rendimientos de $\mathrm{Y}=0,85-0,92 \mathrm{~g} \mathrm{~g}^{-1}$ asociados con 1,5-3,5 g L L de biomasa micótica producidos entre las 36 y 48 h de fermentación. Pandey y Ramachandran (2006) reportaron un estudio comparativo de la cepa R. oryzae para evaluar la producción de acido láctico en SmF y SSF, mostrando que éste último tuvo una nivel de producción mayor y los rendimiento fueron de 93,8 y 137,0 $\mathrm{g} \mathrm{L}^{-1}$ en SmF y SSF, respectivamente.

Nuevos reactores se han propuesto para reducir el efecto inhibitorio del metabolito a altas concentración, aumentar la productividad y el rendimiento; como el reactor continuo "Specialized Function wo-Stage Reactor" (SFTS) diseñado por Amrane y Prigent (1996) en el cual se mejora la productividad volumétrica en una segunda etapa, el "Membrana CellRecycle Bioreactor" (MCRB) por Xu et al. (2006) para obtener alta densidad celular, Giorno et al. (2002) estudiaron el sistema, y Choudhury y Swaminathan (2006) lo usaron alimentando sustrato a 100 y $120 \mathrm{~g} \mathrm{dm}^{-3}$ y obteniendo una máxima densidad celular de $145 \mathrm{~g} \mathrm{dm}^{-3}$ y productividad de $34 \mathrm{~g} \mathrm{dm}^{-3} 1 \mathrm{~h}^{-1}$. El "Stirred Ceramic Membrane Reactor" (SCMR) propuesto por Ohashi et al. (1999) establece un sistema que provee un proceso continuo de bajo costo a partir de sustratos baratos.

Recientes estudios, utilizan la Metodología de Superficie de Respuesta (MSR) para evaluar el efecto de las diferentes fuentes de carbono y de nitrógeno en la producción de ácido láctico, con el objeto de desarrollar procesos eficientes y económicos en el laboratorio que involucren la selección de materias primas renovables de bajo costo por fuentes costosas, tal como, lo reportado por Altaf et al. (2007a) que sustituyó completamente peptona y extracto de levadura comercial por lenteja roja y levadura de panadería en un sistema sumergido de fermentación.

La MSR puede identificar y cuantificar las interacciones entre parámetros y ha sido 
extensamente aplicada para optimizar condiciones de los medios de cultivo y otros parámetros del proceso (Gündüz 2005). Las metodologías usadas para determinar los parámetros óptimos de producción de ácido láctico se encuentran basadas en la metodología de Taguchi (Nagarjun et al. 2005; Bhatta y Srivastava 2008; Korbekandi et al. 2007), Plackett-Burman (Altaf et al. 2007b; Patel et al. 2007), Diseño Central Compuesto (Altaf et al. 2007a; De Lima et al. 2009).

\section{CONCLUSIONES}

El ácido láctico puede ser obtenido por vía biotecnológica a partir de derivados de recursos renovables como azúcar, melaza, lactosuero, materiales amiláceos y lignocelulósicos, almidones, yuca, licor de maíz. Muchas investigaciones explican los distintos factores que controlan la fermentación para producir ácido láctico, desde los microorganismos implicados como las Bacterias Ácido Lácticas, rendimiento del proceso, sistemas batch, fedbatch y continuo, necesidades nutricionales $y$ suplementos. Sin embargo, las investigaciones van dirigidas a producir ácido láctico con el desarrollo de microorganismos de alto rendimiento y la reducción del costo de la materia prima.

\section{REFERENCIAS}

Adsul, M., Khire, J., Bastawde, K. y Gokhale, D. 2007. Production of Lactic Acid from Cellobiose and Cellotriose by Lactobacillus delbrueckii Mutant Uc-3. Applied and Environmental Microbiology 73 (15): 5055-5057.
Agarwal, L., Dutt, K., Meghwanshi, G. y Saxena, R. 2008. Anaerobic fermentative production of lactic acid using cheese whey and corn steep liquor. Biotechnology Letters 30 (4): 631-635.

Alonso, J., Garrote, G., Domínguez, H., Santos, V. y Parajo, J. 2009. Lactic acid from apple pomace: a laboratory experiment for teaching valorisation of wastes. CyTA - Journal of Food 7 (2): 83 - 88.

Altaf, M., Naveena, B. y Gopal, R. 2005. Screening of Inexpensive Nitrogen Sources for Production of $\mathrm{L}(+)$ Lactic Acid from Starch by Amylolytic Lactobacillus amylophilus GV6 in Single Step Fermentation. Food Technology and. Biotechnolology 43 (3): 235-239.

Altaf M., Naveena, B., Venkateshwar, M., Vijay Kumar, E. y Gopal, R. 2006. Single step fermentation of starch to $L(+)$ lactic acid by Lactobacillus amylophilus GV6 in SSF using inexpensive nitrogen sources to replace peptone and yeast extract-optimization by RSM. Process Biochemistry 41(2): 465-472.

Altaf, M., Naveena, B. y Gopal, R. 2007a. Use of inexpensive nitrogen sources and starch for $\mathrm{L}(+)$ lactic acid production in anaerobic submerged fermentation. Bioresource Technology 98: 498-503.

Altaf, M., Venkateshwar, M., Srijana, M. y Gopal, R. 2007b. An economic approach for L-(+) lactic acid fermentation by Lactobacillus amylophilus GV6 using inexpensive carbon and nitrogen sources. 
Journal of Applied Microbiology 103(2): $372-380$.

Altiok, D. 2004. Kinetic Modelling of Lactic Acid Production from Whey. Master of Science, Izmir Institute of Technology, Food Engineering, Izmir, Turkey.

Amrane, A. y Prigent, Y. 1996. A novel concept of bioreactor: Specialized function two-stage continuous reactor, and its application to lactose conversion into lactic acid. Journal of Biotechnology 45 (3): 195-203.

Arasaratnam, V., Senthuran, A. y Balasubramaniam, K. 1996. Supplementation of whey with glucose and different nitrogen sources for lactic acid production by Lactobacillus delbrueckii. Enzyme and Microbial Technology 19 (7): 482-486.

Arias, M., Mariohenao, L., Castrillón, Y. 2009. Producción de ácido láctico por fermentación de mucílago de café con Lactobacillus bulgaricus NRRL-B548. Dyna 76 (158): 147-153.

Bhatta, S. y Srivastava, S. 2008. Lactic Acid Production from Cane Molasses by Lactobacillus delbrueckii NCIM 2025 in Submerged Condition: Optimization of Medium Component by Taguchi DOE Methodology. Food Biotechnology 22 (2): 115 - 139.

Büyükkileci, A. y Harsa, S. 2004. Batch production of $\mathrm{L}(+)$ lactic acid from whey by Lactobacillus casei (NRRL B-441).
Journal of Chemical Technology and Biotechnology 79: 1036-1040.

Bustos, G., De la Torre, N., Martínez, M., Casares, A. y Domarco, Y. 2004. Evaluación de azúcares hemicelulósicos de las podas de sarmiento y lías de vinificación como medio nutritivo para la producción de ácido láctico por Lactobacillus pentosus. Ciencia y Tecnología Alimentaria 4 (004): 283-291.

Choudhury, T. y Swaminathan, T. 2006. Lactic acidfermentation in cell-recyclemembrane bioreactor. Applied Biochemistry and Biotechnology 128 (2): 171-183

Costa, V., Basso, T., Angeloni, L. y Marilia Oetterer, L. 2008. Production of acetic acid, ethanol and optical isomers of lactic acid by Lactobacillus strains isolated from industrial ethanol fermentations Ciência e Agrotecnologia 32 (2): 503-509.

De Lima, C., Fontes, L., Blanco, K. y Contiero, J. 2009. Response surface optimization of $\mathrm{D}(-)$-lactic acid production from LactobacillusSMI8 using corn steep liquor and yeast autolysate as nitrogen sources. African Journal of Food Science 3 (9): 257-261.

Ding, S. y Tan, T. 2006. L-lactic acid production by Lactobacillus casei fermentation using different fed-batch feeding strategies. Process Biochemistry 41 (6): 1451-1454.

Dong-Mei Bai, Min-Ze Jia, Xue-Ming Zhao, Rui Ban, Fei Shen, Xin-Gang Li, Shi-Min Xu. 2003. $L(+)$ lactic acid production 
by pellet-form Rhizopus oryzae R1021

in a stirred tank fermentor. Chemical Engineering Science 58 (3-6): 785-791.

Dumbrepatil, A., Adsul, M., Chaudhari, S. y Jayant Khire, A. 2008. Utilization of Molasses Sugar for Lactic Acid Production by Lactobacillus delbrueckii subsp. delbrueckii Mutant Uc-3 in Batch Fermentation. Applied and Environmental Microbiology 74 (1): 333-335.

Giorno, L., Chojnacka, K., Donato, L. y Drioli, E.2002. Study of a Cell-Recycle Membrane Fermentor for the Production of Lactic Acid by Lactobacillus bulgaricus. Industrial and Engineering Chemistry Research 41 (3) : 433-440.

Göksungur, Y., Gündüz, M. y Harsa, S. 2005. Optimization of lactic acid production from whey by L. casei NRRL B-441 immobilized in chitosan stabilized Caalginate beads. Journal of Chemical Technology and Biotechnology 80(11): 1282-1290.

Gopal, R., Altaf, Md., Naveena, B., Venkateshwar, M. y Vijay Kumar E. 2008. Amylolytic bacterial lactic acid fermentation -A review. Biotechnology Advances 26 (1): 22-24. Escrita donde no debe ir

Gündüz, M. 2005. Lactic acid production by Lactobacillus casei NRRL B-441 immobilized in chitosan stabilized Ca-alginate beads. Tesis de Master of Science, Izmir Institute of Technology, Izmir.
Hofvendahl, K. y Hägerdal, B. 2000. Factors affecting the fermentative lactic acid production from renewable resources. Enzyme and Microbial Technology 26(24): 87-107.

Huang, L., Jin, B., Lant, P. y Zhou, J. 2005. Simultaneous saccharification and fermentation of potato starch wastewater to lactic acid by Rhizopus oryzae and Rhizopus arrhizus. Biochemical Engineering Journal 23(3): 265-76.

Idris, A. 2006. Effect of sodium alginate concentration, bead diameter, initial $\mathrm{pH}$ and temperature on lactic acid production from pineapple waste using immobilized Lactobacillus delbrueckii. Process Biochemistry 41 (5): 1117-1123.

Joshi, S., Singhvi, M., Khire, J. y Gokhale, D. 2010. Strain improvement of Lactobacillus lactis for D-lactic acid production. Biotechnology Letters 32 (4): 517-520.

Kenji Okano, S., Sakurako, K., Junya, N., Hideki, F. y Akihiko, K. 2007. Improvement in lactic acid production from starch using $\alpha$-amylase-secreting Lactococcus lactis cells adapted to maltose or starch. Applied Microbiology and Biotechnology 75 (5): 1007-1013.

Korbekandi, H., Abedi, D., Jalali, M., Fazeli, M. y Heidari, M. 2007. Optimization of Lactobacillus casei growth and lactic acidproduction in batch culture.Journal of Biotechnology 131(1): 133-187. 
Kourkoutas, Y., V. Xolias, V., Kallis,M., Bezirtzoglou, E. y Kanellaki, M. 2005. Lactobacillus casei cell immobilization on fruit pieces for probiotic additive, fermented milk and lactic acid production. Process Biochemistry 408(1): 411-416.

Kyla“-nikkila, K., Hujanen, M., Leisola, M . y Palva, A. 2000. Metabolic Engineering of Lactobacillus helveticus CNRZ32 for Production of Pure L-(1)-Lactic Acid. Applied and Environmental Microbiology 66 (9): 3835-3841.

Lee, K. 2004. Comparison of fermentative capacities of Lactobacilli in single and mixed culture in industrial media. Process Biochemistry40 (5): 1559-1564.

Limin Wang, Bo Zhao, Bo Liu, Bo Yu, Cuiqing Ma, Fei Su, Dongliang Hua, Qinggang Li, Yanhe Ma, Ping Xu. 2010a. Efficient production of I-lactic acid from corncob molasses, a waste by-product in xylitol production, by a newly isolated xylose utilizing Bacillus sp. strain, Bioresource Technology, 101(20): 7908-7915.

Limin Wang, Bo Zhao, Bo Liu, Chunyu Yang, Bo Yu, Qinggang Li, Cuiqing Ma, Ping Xu, Yanhe Ma. 2010b. Efficient production of I-lactic acid from cassava powder by Lactobacillus rhamnosus, Bioresource Technology, 101(20): 7895-7901.

Liu, Y., Wen, Z., Liao, W., Liu, C. y Chen, S. 2005. Optimization of the process for the production of $\mathrm{I}(+)$-lactic acid from cull potato by Rhizopus oryzae. Engineering in Life Sciences 5(4): 343-349.
Marja Ilmén, K., Koivuranta, K., Ruohonen, L., Suominen, P. y Penttilä, M. 2007. Efficient Production of L-Lactic Acid from Xylose by Pichia stipitis. Applied and Environmental Microbiology 73 (1): 117-123.

Marták, J., Schlosser, S., Sabolova, E., Kristofi'kova, L. y Rosenberg, M. 2003. Fermentation for lactic acid with Rhizopus arrhizus in a stirred tank reactor with a periodical bleed and feed operation. Process Biochemistry 38(11): 1573-1583.

Min-Tian, G., Koide, M., Gotou, R., Takanashi, H., y Hirata, M. 2005. Development of a continuous electrodialysis fermentation system for production of lactic acid by Lactobacillus rhamnosus. Process Biochemistry 40(3-4): 1033- 1036.

Mirdamadi, S., Atashgahi, S., Rajabi, A., AzizMohseni, F., Roayaei, M. y Hamedi, J. 2008. Cell entrapment of Lactobacillus casei subsp. casei ATCC 39392 for lactic acid production. Iranian Journal of Biotechnology 6 (1): 16-21.

Moo-Young, M., Moreira, A. y Tengerdy, R. 1983 Principles of the Solid Substrate Fermentation. In Filamentous Fungi. 4. Ed. J. E. Smith, D. R. Berry and B. Kristianse, Edward Arnold, p117-144.

Mussatto, S., Fernandes, M., Mancilha, I. y Roberto, I. 2008. Effects of medium supplementation and $\mathrm{pH}$ control on lactic acid production from brewer's spent grain. Biochemical Engineering Journal 40 (3): 437-444. 
Nabi, B. y Ardalan, B. 2004. Batch and continuous production of lactic acid from whey by immobilized Lactobacillus. Journal of Environmental Studies 30(34): 47-53.

Nagarjun, P., Rao, R., Rajesham, S. y Rao, L. 2005. Optimization of Lactic Acid Production in SSF by Lactobacillus amylovorus NRRL B-4542 Using Taguchi Methodology. The Journal of Microbiology 43 (1): 38-43.

Nancib, A., Nancib, N., Meziane-Cherif,D., Boubendir,A., Fick,M. y Boudrant, J. 2005. Joint effect of nitrogen sources and B vitamin supplementation of date juice on lactic acid production by Lactobacillus casei subsp. rhamnosus. Bioresource Technology 96 (1): 63-67.

Narayanan, N., Roychoudhury,P.K.,ySrivastava, A. 2004. $L(+)$ lactic acid fermentation and its product polymerization. http:// www.ejbiotechnology.info/content/ vol7/issue2/full/7/index.html. Electronic Journal of Biotechnology 7 (2).

Naveena,B., Altaf, Md., Bhadrayya, K., Madhavendra, S. y Gopal, R. 2005a. Direct fermentation of starch to $\mathrm{L}(+)$ lactic acid in SSF by Lactobacillus amylophilus GV6 using wheat bran as support and substrate-medium optimization using RSM. Process Biochemistry 40(2): 681690.

Naveena, B., Altaf, M., Bhadrayya, K., y Madhavendra, S. 2005b. Selection of medium components by Plackett-Burman design for production of $\mathrm{L}(+)$ lactic acid by Lactobacillus amylophilus GV6 in SSF using wheat bran. Bioresource Technology 96 (4): 485-490.

Oh, H., Wee, Y., Yun, Y., Han, S., Jung, S. y Ryu, H. 2005. Lactic acid production from agricultural resources as cheap raw materials. Bioresource Technology 96 (13): 1492-1498.

Ohashi, R., Yamamoto, T. y Suzuki, T. 1999. Continuous production of lactic acid from molasses by perfusion culture of Lactococcus lactis using a stirred ceramic membrane reactor. Journal of Bioscience and Bioengineering 87 (5): 647-654.

Ohkouchi, Y. y Inoue Y. 2006. Direct production of $\mathrm{L}(+)$-lactic acid from starch and food wastes using Lactobacillus manihotivorans LMG18011. Bioresource Technology 97: 1554-1562.

Okafor, N. 2007. Modern Industrial Microbiology and Biotechnology. Enfield, New Hampshire, United States of America: Science Publishers.

Okano, K., Kimura, S., Narita, J., Fukuda, H. y Kondo, A. 2007. Improvement in lactic acid production from starch using $\alpha$-amylase-secreting Lactococcus lactis cells adapted to maltose or starch. Applied MicrobiolBiotechnology 75(5):1007-13.

Pandey, A. y Ramachandran, S. 2006. Process Developments in Solid-State Fermentation for Food Applications. In: 
Shetty, K., Paliyath, G., Paliyath, P. y R. Levin, Food Biotechnology. Boca Raton, p109-133.

Pandey, A. 1992. Recent process developments in solid state fermentation. Process Biochem., 27, 109-17.

Panesar, P., Kennedy, J. y Knill, C. 2007a. Applicability of pectate-entrapped Lactobacillus casei cells for $\mathrm{L}(+)$ lactic acid production from whey. Applied Microbial Biotechnology 74(1): 35-42.

Panesar, P., Kennedy, J., Gandh, D. y Bunko, K. 2007b. Bioutilisation of whey for lactic acid production. Food Chemistry 105(1): 1-14.

Park, E., Kosakai, Y. y Okabe, M. 1998. Efficient production of $\mathrm{L}(+)$-lactic acid using mycelial cotton-like flocs of Rhizopus oryzae in an air-lift bioreactor. Biotechnology Progress 14(5): 699-704.

Patel, K. 2007. Statistical screening of medium components by Plackett-Burman design for lactic acid production by Lactobacillus sp. KCP01 using date juice. Bioresource Technology 98 (1): 98-103.

Patil, S., Kadam, S., Bastawde, K., Khire, J. y Gokhale, D. 2006. Production of lactic acid and fructose from media with cane sugar using mutant of Lactobacillus delbrueckii NCIM 2365. Letters in Applied Microbiology 43 (1): 53-7.

Pessôa de França, F., Auta, M. y Santos, F. 2009. Enhancement of lactic acid fermentation by Lactobacillus delbrueckii ATCC 6949 using sugarcane molasses. Canadian Journal of Pure and Applied Sciences 3 (2): 773-778.

Plessas, S., Bosnea, L., Psarianos, C., Koutinas, A., Marchant, R. y Banat, I. 2008. Lactic acid production by mixed cultures of Kluyveromyces marxianus, Lactobacillus delbrueckii ssp. bulgaricus and Lactobacillus helveticus. Bioresource Technology 99 (13) 5951-5955.

Qi, B. y Yao, R. 2007. L-lactic acid production from lactobacillus casei by solid state fermentation using rice straw. BioResources 2 (3): 419-429.

Rivas, B., Moldes, A. y Domínguez, J. 2004a. Development of culture media containing spent yeast cell of Debaryomyces hanseii and corn step liquour for lactic acid production with Lactobacillus rhamnosus. Internacional Journal of Food Microbiology 97: 93-98.

Rivas, B., Moldes, A. y Domínguez, J. 2004b. Lactic acid production from corn cobs by simultaneous saccharification and fermentation: a mathematical interpretation. Enzyme and Microbial Technology, 34 627-634.

Rojan, P., Madhavan, K., Syamaprasad, A. y Pandey, A. 2005. $\mathrm{L}(+)$-lactic acid production using Lactobacillus casei in solid-state fermentation. Biotechnological Letter 27: 1685-8.

Rojan, P., Madhavan, K., Syamaprasad, A. y Pandey, A. 2006. Solid-state fermentation 
for L-lactic acid production from agro wastes using Lactobacillus delbrueckii. Process Biochemistry 41(4): 759-763.

Rojan, P., Madhavan, N. y Pandey, A. 2006a. Simultaneous saccharification and fermentation of cassava bagasse for L (+) lactic acid production using Lactobacilli. Applied Biochemistry and Biotechnology134(3): 263-72.

Rojan, P., Madhavan, N. y Pandey, A. 2006 b. Simultaneous saccharification and L-(+)lactic acid fermentation of protease treated wheat bran using mixed culture of Lactobacilli. Biotechnological Letter 28(22):: 1823-1826.

Rojan, P., Madhavan, N. y Pandey, A. 2006c. Solid-state fermentation for L-lactic acid production from agro wastes using Lactobacillus delbrueckii. Process Biochemistry 41(3): 759-63.

Rojan, J., Nampoothiri, K. y Pandey, A. 2007. Fermentative production of lactic acid from biomass: an overview on process developments and future perspectives. Applied Microbiology and Biotechnology 74(3): 524-34.

Rojan, P., Anisha, G., Madhavan, K. y Pandey, A. 2009. Direct lactic acid fermentation: Focus on simultaneous saccharification and lactic acid production. Biotechnology Advances 27(2): 145-152.

Sakai, K., Fujii, N. y Chukeatirote, E. 2007. Racemisation of L-lactic acid in $\mathrm{pH}$-swing open fermentation of kitchen refuse by selective proliferation of Lactobacillus plantarum. Journal of Bioscience and Bioengeering 102(3): 227-232.

Schepers, A., Thibault, J. y Lacroix, C. 2002. Lactobacillus helveticus growth and lactic acid production during $\mathrm{pH}$-controlled batch cultures in whey permeate/yeast extract medium. Part I. Multiple factor kinetic analysis. Enzyme and Microbial Technology 30(2): 176-186.

Schepers, A., Thibault, J. y Lacroix, C. 2006. Continuous lactic acid production in whey permeate/yeast extract medium with immobilized Lactobacillus helveticus in a two-stage process: Model and experiments. Enzyme and Microbial Technology 38(3-4): 324-337.

Serna, C. y Rodriguez, A. 2005. Produccion biotecnologica de ácido láctico: estado del arte. Ciencia y Tecnologia de Alimentos 5(1): 54-65.

Serna, L. y Rodríguez, A. 2006. Lactic acid production by a strain of Lactococcus lactis subs lactis isolated from sugar cane plants. Electronic Journal of Biotechnology.http://www. ejbiotechnology.info/content/vol9/ issue1/full/10/. [Abril de 2010].

Serna, L. y Rodríguez, A. 2007. Lactic Acid Fermentative Production using Waste from the Harvest of Green Sugar Cane as a Substrate. Interciencia 32 (005): 328-332.

Serrato, B. y Caicedo, M. 2005. Efecto de la concentración de material inerte 
en un biocatalizador de alginato de calcio con células inmovilizadas sobre la fermentación láctica. Ingeniería e Investigación 25 (2): 78-82.

Shen, X., y Xia, L. 2006. Lactic acid production from cellulosic waste by immobilized cells of Lactobacillus delbrueckii. World Journal of Microbiology and Biotechnology 22(11): 1109-1114.

Sule, B., Murat, E. y Dursun, O. 2004. Effect of diferent carbone sources on $\mathrm{L}(+)$ lactic acid production by Rhizopus oryzae. Biochemical Engineering Journal 21(1): 33-7.

Tanaka, T., Hoshina, M., Tanabe, S., Sakai, K., Ohtsubo, S. y Taniguchi, M. 2005. Production of D-lactic acid from defatted rice bran by simultaneous saccharification and fermentation. Bioresource Technology 97(2): 211-217.

Tay, A. y Yang, S. 2002. Production of L(+)lactic acid from glucose and starch by immobilized cells of Rhizopus oryzae in a rotating fibrous bed bioreactor. Biotechnoogy. Engineering 80(1): 1-12.

Thomsen, M., Guyot, J., Kiel, P. 2007. Batch fermentations on synthetic mixed sugar and starch medium with amylolytic lactic acid bacteria. Applied Microbiology and Biotechnology 74(3): 540-6.

Urríbarrí, L., Vielma, A., Paéz, G., Ferrer, J. y Mármol, Z. 2004. Producción de acido láctico a partir de suero de leche utilizando Lactobacillus helveticus en cultivo continuo. Revista Cientifica de la
Facultad de Ciencias Veterinarias de la Universidad del Zulia 14: 297-302.

Vasala, A., Panula, J., Neubauer, P. 2005. Efficient lactic acid production from high salt containing dairy byproducts by Lactobacillus salivarius ssp salicinius with pre-treatment by proteolytic microorganisms. Journal of Biotechnology 117(4): 421-431.

Vázquez, J. 2008. Mathematical tools for objective comparison of microbial cultures Application to evaluation of 15 peptones for lactic acid bacteria productions. Biochemical Engineering Journal 39(2): 276-287.

Vijayakumar, J., Aravindan, R. y Viruthagiric, T. 2008. Recent Trends in the Production, Purification and Application of Lactic Acid. Chemical and Biochemical Engenniering 22(2): 245-264.

Wang, Q., Wang, X., Wang, X., Ma, H. y Ren, N. 2005. Bioconversion of kitchen garbage to lactic acid by two wild strains of Lactobacillus species. Journal of Enviromental Science and Health 40(10): 1951-62.

Wee, Y., Kim, J. y Ryu, H. 2006a. Biotechnological Production of Lactic Acid and Its Recent Applications. Food Technology and Biotechnology 44(2): 163-172.

Wee, Y.-J. Kim, H.-O. Yun, J.-S. Ryu, H.-W. 2006b. Pilot-Scale Lactic Acid Production via Batch Culturing of Lactobacillus 
sp. RKY2 Using Corn Steep Liquor as a Nitrogen Source. Food Technology Biotechnology44 (2): 293-29.

Wee, Y., Reddy, L. y Ryu, H. 2005. Fermentative production of $\mathrm{L}(+)$-lactic acid from starch hydrolyzate and corn steep liquor as inexpensive nutrients by batch culture of Enterococcus faecalis RKY1. Journal of Chemical Technology y Biotechnology 83 (10): 1387 - 1393.

Xu, G., Chu, J., Wang, Y., Zhuanga Y., Zhang, S. y Peng, H. 2006. Development of a continuous cell-recycle fermentation system for production of lactic acid by Lactobacillus paracasei. Process Biochemistry 41 (12): 2458-2463.

Yu MC, Wang RC, Wang CY, Duan KJ, Sheu DC. 2007. Enhancedproduction of $\mathrm{L}(+)$-lactic acid by floc-form culture of
Rhizopus oryzae.J. Chinese Inst. Chem. Eng. 38: 223-228.

Zhang, Z., B. Jin, B. y Kelly, J. 2005. Production of lactic acid and byproducts from waste potato starch by Rhizopus arrhizus : role of nitrogen sources. World Journal of Microbiology and Biotechnology 23 (2): 265.

Zhang, Z., Jin, B. y Kelly, J. 2007a. Production of lactic acid from renewable materials by Rhizopus fungi. Biochemical Engineering Journal 35: 251-263.

Zhang, Z., Jin, B. y Kelly, J. 2007b. Production of lactic acid and byproducts from waste potato starch by Rhizopus arrhizus: role of nitrogen sources. Journal of Microbiology and Biotechnology 23 (2): 229-236. 